\title{
A physical and numerical based model for early prediction of landslides using wireless sensor network
}

\author{
Vinay Kumar Singh \\ 1 Department of Earth Science, IIT Bombay, Powai, 211002, India \\ Corresponding author: vinayrajput@iitb.ac.in
}

\begin{abstract}
Landslides are a frequent and recurrent problem in hilly regions of India and predicting them is always a challenging task. In this paper, an attempt was made to deal with this problem using advanced physical and numerical modeling methods. Detailed understanding of the initial slope failures is very interesting, and challenging at the same time, in the design and development of wireless sensor network based on early warning of landslide monitoring. A small scale physical model was developed to assess the instability through a sensor network with variable rain fall intensity. This was achieved by increasing the simulated rain water flow intensity in different time spans (dry condition, at $\mathrm{t}=0$ to $\mathrm{t}=30 \mathrm{~min}, 0.5 \mathrm{~mm} / \mathrm{min}$ at $\mathrm{t}=30 \mathrm{to} \mathrm{t}=60 \mathrm{~min}, 0.75 \mathrm{~mm} / \mathrm{min}$ at $\mathrm{t}=60 \mathrm{to} \mathrm{t}=$ $91 \mathrm{~min}$ and $1 \mathrm{~mm} / \mathrm{min}$ at $\mathrm{t}=91$ to $\mathrm{t}=120 \mathrm{~min}$ ). The water level and movement in the slope was recorded by rainfall sensor, vibration sensor, soil moisture sensor and a digital camera. The following changes were observed during the slope failure: a) movement of small particles at top of the slope; b) initial failure of medium size soil particle; c) scouring of soil mass; d) whole slope collapse. The obtained results clearly indicated the superiority and effectiveness of the proposed system in providing a factor of safety for the progressive slope.
\end{abstract}

Keywords: Landslides; Factor of Safety (FoS), Wireless Sensor Network; Slope Stability

\section{Introduction}

Shallow landslides/soil slips are common in different geo-environmental conditions and have caused significant damages around the world. Soil slips are surficial portions of the soil which results from rainfall detach from shallow depths of the slope and move in different ways (Montrasio et al. 2015). Shallow landslides frequently occur during monsoons in a continuous sequence of wetting, heating and drying (Bogena et al 2007, Shukla et al. 2014). Landslides are investigated and identified by typical slope stability analysis (Bogena et al 2007). There are several factors and parameters that indicate or trigger landslides and must be studied before site selection for any monitoring or development activity. Such factors include weather conditions, seismic movement, rate of rainfall, wind speed, and groundwater level. Landslides are frequent in steep slopes and are further aggravated due to anthropogenic activities; however, this also arise in gentle slopes make them challenging to recognize (Corominas et al. 2014). Their capability cause extensive damage make landslides an tremendously significant issue of research and investigation.

Early slope failure monitoring can deliver valuable information with regards to its dynamics of landslide and could save lives and prevent damage of properties in case of slope failures (Vishal et al 2017, Gui et al. 2011). Bláha and Müller (2008) suggested that geophysical methods are useful for initial stages of landslides monitoring. Previously, a number of physical model based shallow landslides monitoring system have been proposed; these use factor of safety for slope stability analysis (Hodge et al. 2015, Baum, et al 2015). In addition, there are simplified slope stability models which utilize the physical and mechanical properties of the material for slope monitoring (Corominas 
et al. 2014). Along with these advancements, several laboratory scale experiments have been conducted to develop better understanding of failure mechanisms and conditions that lead to shallow landslides. Lepore et al. (2013) proposed a laboratory scale facility for shallow landslide monitoring in sandy soil and observed the development of water pore pressure and initiation of failures. They studied the effect of initial density, grain size and fine particle content to see the mobility of intense rain fall induced shallow landslides. A few studies with a similar approach but considering other aspects of shallow landslides, such as viz. initiation, depth of failure, mode and sediment discharge, have also been attempted (Vishal et al 2015, Singh et al. 2016). Recent works have also focused on advanced simulations of slopes for analysis and prediction of failures in natural and engineered slopes (Pradhan et al 2013). Ramesh (2014) developed a wireless sensor network-based landslide monitoring and forecasting system for predicting the probability of landslides occurrences and generate alert. The system was validated by generating real time alerts during heavy rainfall during the monsoons of July 2009. The system is able to issue an alert to the local community and save lives and property as well as allowing the administration to plan the execution and sheltering. The proposed landslides monitoring system is useful for all categories of landslides. However, a high number of sensors are required in the system, which leads to manpower requirements, higher system cost and difficulty in field deployment of sensor nodes, since these nodes are comparatively bigger (Ramesh 2014). The main aim of this study was to find out appropriate sensors and to incorporate some new intelligent techniques that can be added for the betterment of the simulated system and eventually develop a compact and energy efficient landslides monitoring and prediction system.

\section{Background of the study}

To introduce a rainfall based physical model for landslides monitoring, it is required to define the current status of slope. Factor of safety (FoS) is being considered for site specific landslides (Collins and Znidarcic 2014). To analyze the stability of rainfall-based slope failure, FoS could be monitored and retrieved using wireless sensor networks. Development and implementation of rainfall-based landslides monitoring and detection are mainly dependent on the type of the sensors used. It is well defined that the selection of sensors is highly dependent on the landslides triggering factors viz. e.g., heavy rainfall, ground water level moisture, vibration (Rahardjo et al. 2010). In the proposed early warning system, the monitored parameters are rainfall, soil moisture and acceleration at different depths. Several advanced electronic sensors have also been developed for landslide monitoring. Recent technical advancement, in the wireless sensor networks, enable situ sensor-based landslide monitoring which can tolerate higher analysis errors with greater accuracy in monitoring. However, in traditional technologies, analysis errors are relatively low; conversely, sampling errors may be extensive because of the limited sampling intensity. Huisman et al. (2003) discussed wireless sensor network based secluded soil monitoring throughout five months in a real-world environment. Wireless sensors have been extensively utilized in other fields such as environmental engineering, earth dynamics and space and climate science.

ZigBee (IEEE 802.15.4 protocol) is a short range, very low power wireless technology, whose performance properties include low cost, low complexity and self-organizing ability. The maximum data rate for a ZigBee device is $250 \mathrm{Kbps}$ and is easy to implement. ZigBee-based wireless sensor networks are able to support more than 50,000 interconnected nodes. It uses small packets compared to Wi-Fi and Bluetooth and has capability to operate for several months without the need for any battery replacement. ZigBee can automatically establish its network (Lee et al. 2013). Physical obstructions, wireless network interference, network usage and load local environment characteristics are the major uncertainties that affect the performance of the ZigBee based wireless sensor networks. This wireless technology does not achieve high throughputs and small latency at a time. The optimum load size of network should be optimized to maintain optimum value of latency without losing much resources, energy and time, while transmitting the data (Lee et al. 2013). 


\section{Materials and Methodology}

Factors that influence slope deformation during slope failure can be characterized into two parts i.e., fundamental factors and triggering factors. Factors associated with erosion, deterioration of material through water, earthquake and induced forces that lead to slope deformation are known as triggering factors whereas the intrinsic slope factors such as slope size, slope angle, soil strength can be categorized as fundamental factors. In order to interpret the behavior of slope deformation, a physical model of dimension $100 \mathrm{~cm}$ (length) $\times 30 \mathrm{~cm}$ (width) $\times 30 \mathrm{~cm}$ (height) was established as shown in Figure 1. The established physical model slope was monitored through a developed wireless sensor network system to record the rainfall, moisture content and acceleration data with time. The rainfall, i.e., water content in the soil or debris, largely governed the moisture content and acceleration of the soil. Then the numerical simulation for established slope has been done to know the stability and factor of safety of slope for different moisture contents. For that, the mechanical properties (cohesion, friction angle, unit weight and modulus of elasticity etc.,) of the established soil slope of different moisture content are experimented in the lab as per standard. This collaborative study of established soil slope through physical and numerical model may give a better idea to develop a compact and energy efficient landslides monitoring and prediction system.

\subsection{Physical Model ConFigureuration}

The main purpose of this physical model is to reduce the onsite complexity and instability issues and understand the failure characteristics of a typical landslide due to rainfall.The monitoring contains a slope, an angle controller, camera and a heat source. Slope material of different dimensions $(1 \mathrm{~cm} \times \mathrm{b} \mathrm{cm} \times \mathrm{h} \mathrm{cm})$ was placed in the physical model, see Figure 1. The established slope material has been connected to a wireless sensor network system to record the various data's such as moisture, rainfall, humidity and acceleration. Then the established slope was monitored at various rain intensities $(0.5 \mathrm{~mm} / \mathrm{min}, 0.75 \mathrm{~mm} / \mathrm{min}$ and $1 \mathrm{~mm} / \mathrm{min})$ to see the behavior of water fall on slope at different rain intensities. The transportation zone collects the information regarding the movement and deformation of the slope at various water intensities and different angles. The material collection zone records the changes in the size of collected material and traveled distance of the sliding particle or mass.

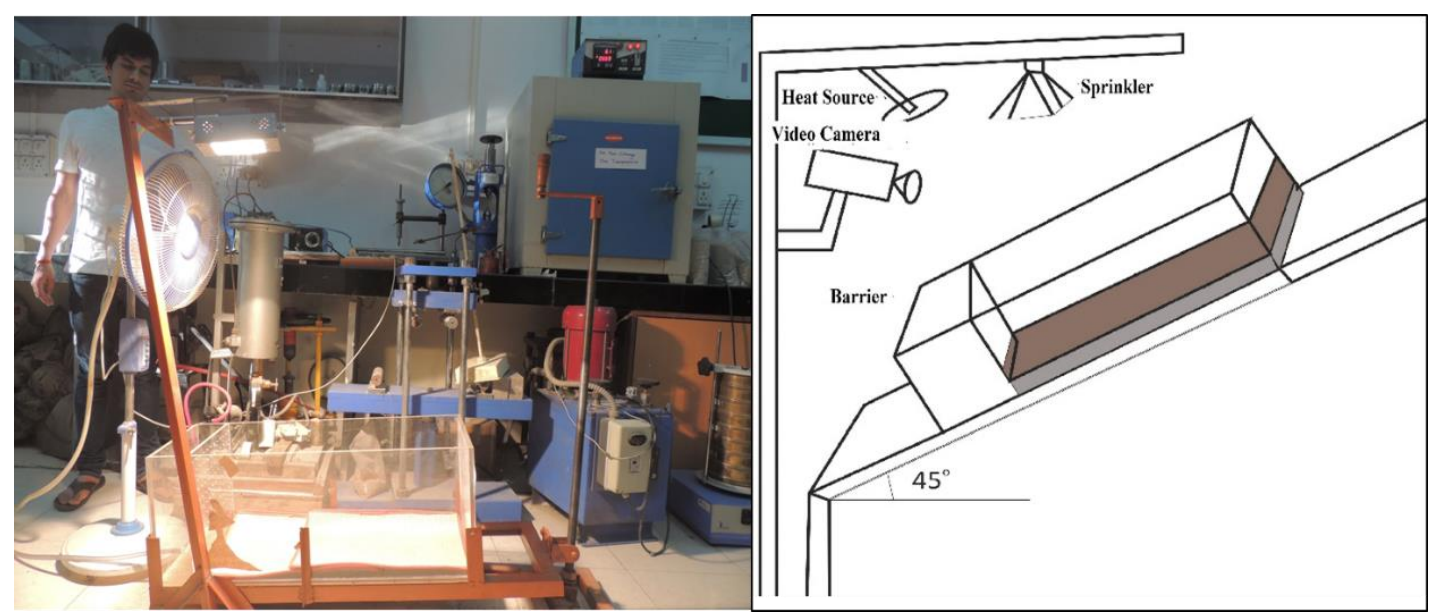

Figure 1. Lab scale physical model for landslide monitoring system. 


\subsubsection{Sensor Deployment}

The aim of this research is to develop a slope monitoring system for earl detection of landslides. A usual landslide prediction system is composed of a wireless sensor network, base station and a remote system. This system will keep track of the movement of the slope and detect pre-failure movement, so that an alarm can be triggered to give warning. The assembled sensor s stem used in this study consists of different sensors to sense parameters such as temperature, moisture, rainfall and acceleration and to determine the threshold values of the individual parameters to anticipate the slope movement.

\subsubsection{Wireless Sensor Network and Early Warning System}

In this study, a laboratory scale physical model capable of monitoring slope failures through wireless sensor networks has been developed. In usual landslide predictions, the system is composed of a wireless sensor network, base station and a remote system. We also created a routing protocol in which, each sensor node "communicate" with their neighboring node and uses multi-hop communications to send the sensed data to the remote station. By using these routing strategies, the energy consumption surely can be reduced, and subsequent development of energy efficient routing protocols will enhance the life-time and coverage area. A wireless sensor network consists of several sensor nodes and each sensor node comprises a processor, a microcontroller, a power supply and an analog-to-digital converter (ADC). The architecture of the sensor network is shown in Figure 2, which shows a combination of temperature, moisture, rainfall and acceleration sensors that can collect pertinent data and can then be interpreted to detect any significant movement in the slope due to individual factor or a combination of parameters. This system will keep track of the movement of the slope and detect the threshold value before failure movement, so that alarm can be triggered to give an early warning. In this wireless system, the radio frequency has been applied to communicate between the sensor node and the remote system.

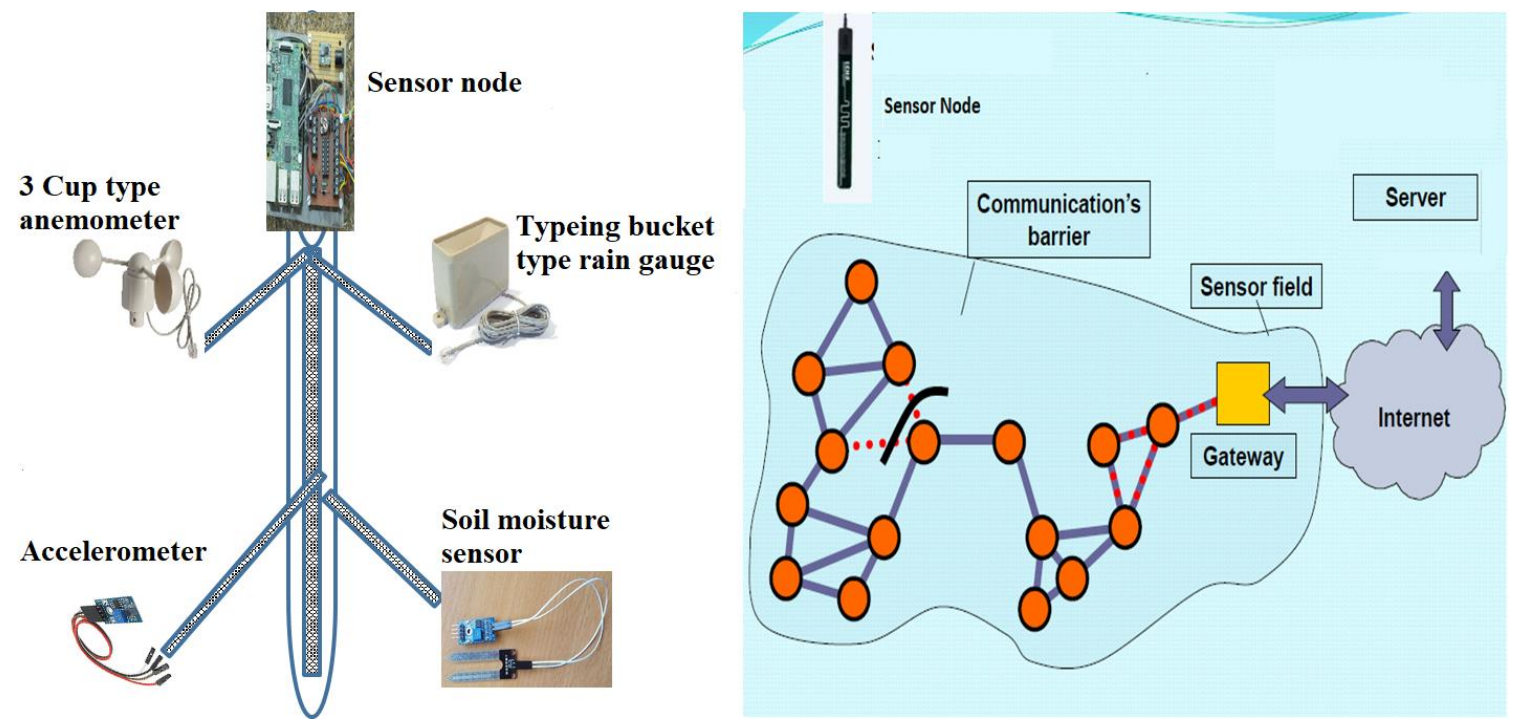

Figure 2. Architecture of the autonomous landslide monitoring system.

\subsubsection{Sensor Node Component}

A general architecture of the sensor node representation is shown in Figure 3. The assembled sensor system used in this study consists of different sensors to sense some parameters such as temperature, moisture, rainfall and acceleration to determine the threshold values of individual parameters to anticipate the slope movement. The microcontroller in the sensor node is one of the key components of the landslides monitoring system. All the peripheral devices viz. sensors, GPS and $\mathrm{Wi}-\mathrm{Fi}$ are connected to it. 


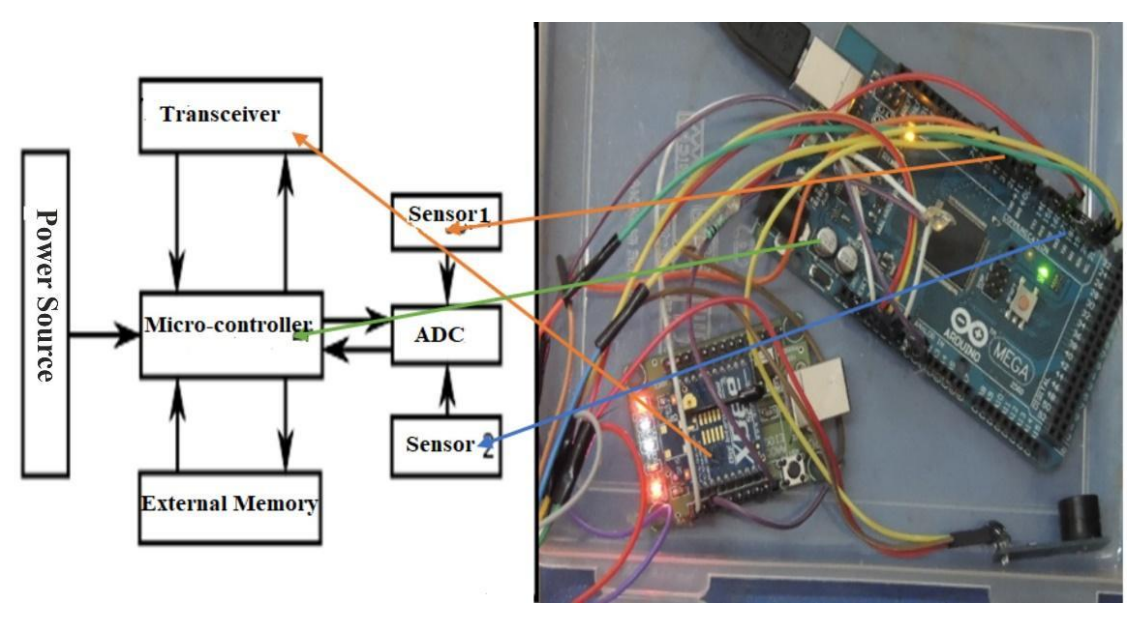

Figure 3. Sensor node representation.

The ARM7 microcontroller receives data from the sensors and transmits it to the remotely located server using a ZigBee wireless module. The ARM7 controller is widely used because of its simplicity, low cost, low power consumption and diversity. The salient features of the controller include a 32-bit ARM7 microcontroller with 64 pin packages. It consists of two 32-bit timers and this also has a chip oscillator operating at $1 \mathrm{MHz}$ to $20 \mathrm{MHz}$. It has a capacity of $500 \mathrm{~Kb}$ on chip flash program memory, having in-system and application programming.

The rain gauge in the sensor node connects and transmits data directly to the network's sink node. The rain gauge is a self-emptying tipping bucket, as illustrated in Figure 4, where each 0.011" $(0.2794 \mathrm{~mm})$ of rain causes one momentary contact closure that can be recorded with a digital counter or microcontroller to interrupt input, where the gauge's switch is attached to the two center conductors of the attached RJ11-terminated cable.

The moisture sensor is used to gather the volume of water from the soil, Figure 4 . The amount of water is an important factor affecting the infiltration of soil for the landslide monitoring and prediction. In unsaturated soils, the volume of water stored within the void spaces is varied, depending on the matric suction within the pore-water. The matric suction is defined as the
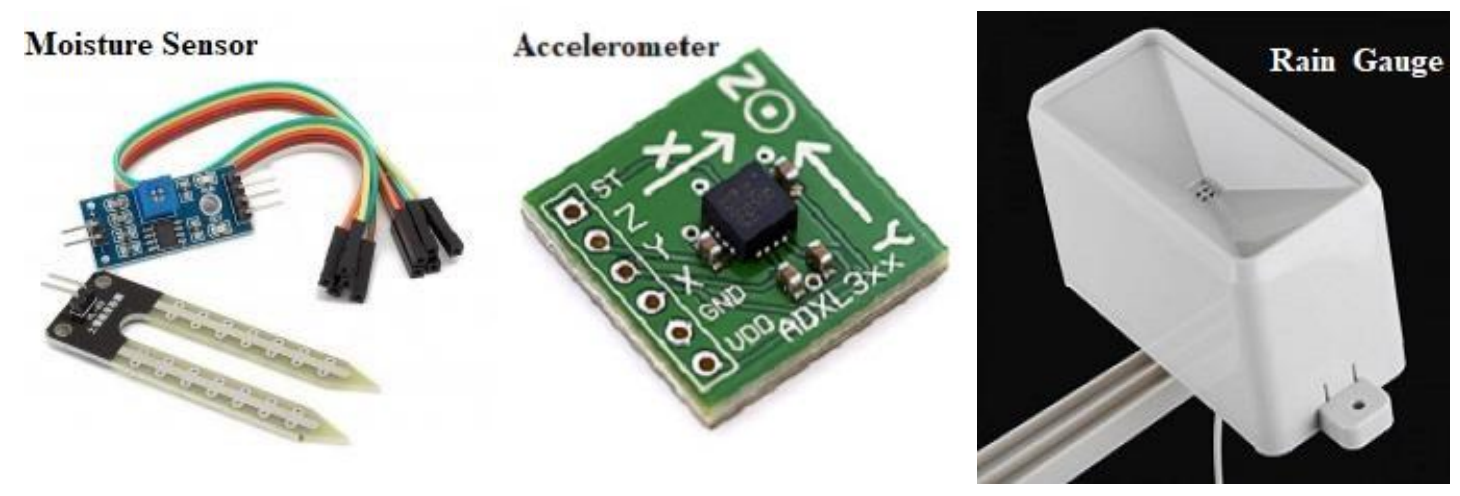

Figure 4. Different sensors in the sensor node.

The difference between the air $\left(u_{\mathrm{a}}\right)$ and water pressure $\left(u_{\mathrm{w}}\right)$. Accordingly, the volume of water content is derived indirectly through measuring the electrical conductivity of the soil medium surrounding the $\mathrm{m}$ using the following equation:

$$
\mathrm{SM}=\left(\mathrm{SM}_{\mathrm{ADC}}-\mathrm{OS} \mathrm{S}_{\mathrm{ADC}}\right) * 200 /\left(1023-\mathrm{OS} \mathrm{SDC}_{\mathrm{ADC}},\right.
$$


where $\mathrm{SM}$ is the soil moisture value (\%), $\mathrm{SM}_{\mathrm{ADC}}$ is the $\mathrm{ADC}$ output and OS $\mathrm{ADC}$ is the offset value at $0 \mathrm{KPa}$.

The accelerometer in the sensor node is used to measure the tilt and vibration with respect to environment situation intensive to landslide monitoring. Thus, the change rate of the velocity and magnitude in 3-axis directions through time can be monitored. However, recent studies point out that micro-electro-mechanical-systems (MEMS)-based accelerometers outperform geophones in terms of power consumption (Hons et al.2008). A MEMS is based on electrochemical vibration sensor for seismic motion monitoring. In Figure 4, the accelerometer, which is an economical MEMS based 3-axis water proof accelerometer for dynamic geo-engineering applications. The acceleration can be calculated using the equation as follows:

$$
\mathrm{ACC}_{\mathrm{i}}=\frac{\mathrm{Sami} /(1024 \dot{\mathrm{x}} \mathrm{R}-\mathrm{Oi})}{\operatorname{Seni}}
$$

where ACC $i$ is the acceleration amount within the direction $i(i=X, Y, Z)$; Sam $i$ is the amount after sampling of axis $i$; $R$ is the voltage reference; $O i$ is the offset, and Sen $i$ is the sensitivity of the accelerometer on axis $i$.

\subsection{Numerical Model Simulation}

The exact geometry of the physical model of established slope was simulated using 2D software to obtain the factor of safety for the different conditions. Numerical simulation has been performed using well validated software Slide V.7, which is based on limit equilibrium method. The limit equilibrium method has been considered most suitable for soil slope stability problem, which has been used by authors to understand the failure mechanism and to estimate the factor of safety (FoS) (Sharma et al., 2017; Singh et al., 2016)

In this method, the loose geomaterials above the trial failure surface is divided into several slices, shown in Figure 5a and 5b). The thickness of all the slices must not to be the same and usually depends on the slope geometry and profile of the geomaterials. Momentum or/and force equilibrium for each slice should be attained.
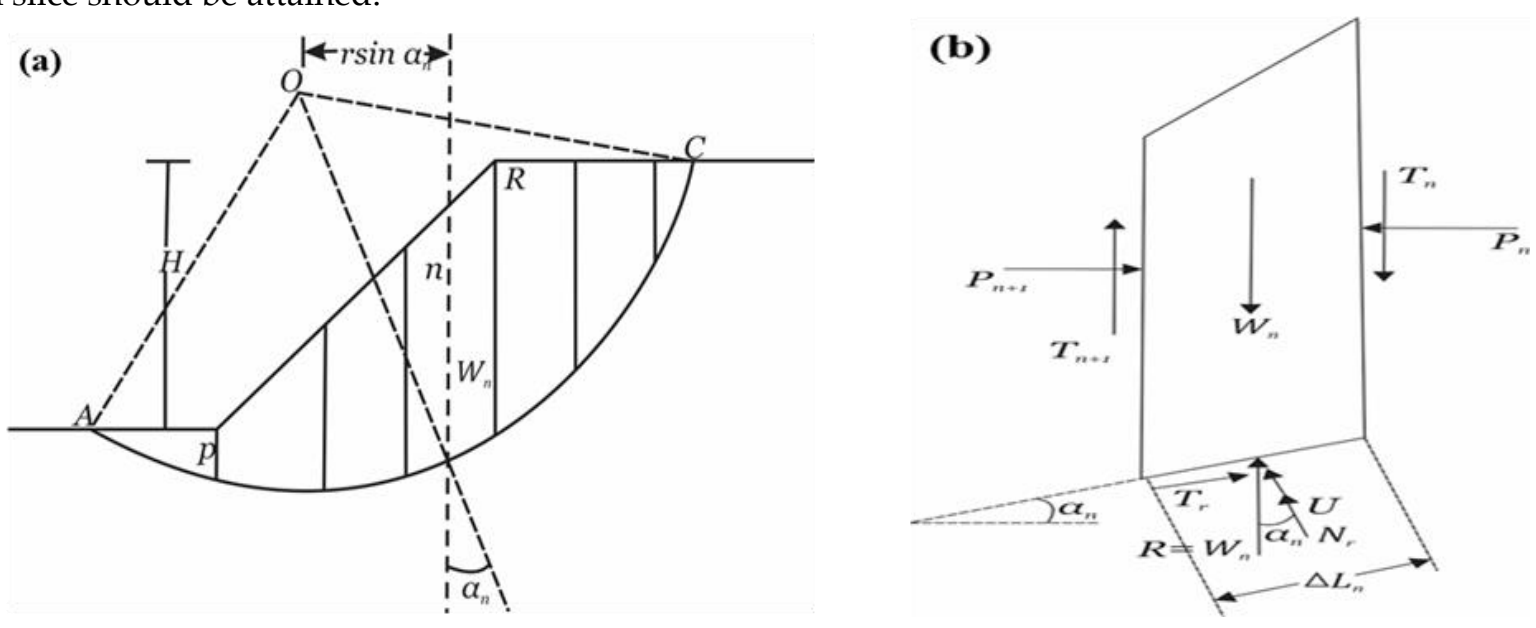

Figure 5. (a) The simplified Bishop's method a slice of the soil above failure plane, (b) Effect of the forces on the side of a particular slide (Bishop, 1955).

The Mohr-Coulomb failure criterion has been used for this particular slope geomaterials and the factor of safety for the established slope has been evaluated by the simplified Bishop method (Bishop 1955). In the above slices, the forces $P_{n}$ and $T_{n}$ have the equal magnitude to the resultant of the $P_{n+1}$ and $\mathrm{T}_{\mathrm{n}+1}$. At equilibrium, forces acting in the vertical direction are: 


$$
N_{r} \cos \alpha_{n}=W_{n}+\left(T_{n}-T_{n+1}\right)-U \cos \alpha_{n}-T_{r} \sin \alpha_{n},
$$

where $\mathrm{W}_{\mathrm{n}}$ is the weight of the slice, $\mathrm{N}_{\mathrm{r}}$ and $\mathrm{T}_{\mathrm{r}}$ are the normal and tangential components, respectively, where $T_{r}=\frac{C \Delta L_{n}}{F_{s}}+N_{r} \frac{\tan \phi}{F_{s}}, \mathrm{~F}_{\mathrm{s}}$ is the (FoS) along the slip surface. After subtitling the value of $\operatorname{Tr}$ in Eq. (3), we get

$$
N_{r}=\frac{W_{n}+\Delta T-U \cos \alpha_{n}+\frac{C \Delta L_{n}}{F_{s}} \sin \alpha_{n}}{\cos \alpha_{n}+\frac{\tan \phi \sin \alpha_{n}}{F_{s}}},
$$

where $\Delta T=T_{n}-T_{n+1}$.

By putting the value $\Delta T=T_{n}-T_{n+1}$ and solving, we obtain

$$
F_{s}=\frac{\sum_{n=1}^{n=p}\left[C \Delta L_{n} \cos \alpha_{n}+\left(\left(W_{n}-U \cos \alpha_{n}\right)+\Delta T\right) \tan \phi\right] \frac{1}{m_{\alpha}}}{\sum_{n=1}^{n=p} W_{n} \sin \alpha_{n}},
$$

where $m_{\alpha}=\cos \alpha_{n}+\frac{\tan \phi \sin \alpha_{n}}{F_{s}}$.

$P_{n}$ and $P_{n+1}=$ normal force act on the side of the slice

$\mathrm{T}_{\mathrm{n}}$ and $\mathrm{T}_{\mathrm{n}+1}=$ shearing force act on the side of the slice

This technique is restricted to the circular type of failure which is given by (Bishop, 1955) and is very useful for the failure assessment of loose type geomaterials viz. soil and derbies.

\section{Results and Discussion}

\subsection{Physical Simulation.}

The model dimensions simulated are $69 \mathrm{~cm} \times 30 \mathrm{~cm} \times 25 \mathrm{~cm}$, where total four parameters viz. rainfall intensity, vibration, moisture and temperature are recorded for small scale sub surface landslides monitoring. The experimental works were performed under the sky, and in natural conditions. The moisture, temperature, wind direction and velocity during experimental work were recorded as: (Temperature $=28^{\circ} \mathrm{C}$, average wind vector (speed) at $11 \mathrm{~km}$ per hour. The wind experienced by any given point in the location is highly dependent on the local topography and other factors, and instantaneous wind speed and directions vary more widely than hourly average.

Different preliminary experiment was performed in order to asses' appropriate slope dimension. In the first experiment, used soil sample was in direct contact with porous stopper, though present solution has been rejected due to highly resistance produced by porous stopper, causing an impractical soil stability condition even there is heavy and long-term rainfall. In order to get extremely negligible disturbance in soil slope, a soil slice was place between the soil sample and stopper. The angle of soil sample is same as physical model to make sure deformation should be within soil sample and stopper. The present was found most suitable for initial investigation purpose and it has been kept same for every experiment.

Simulating the occurrence of preferential flow directions of infiltrating water and using two different rainfall inputs. During each test, the failure mode was observed and the 
triggering time was measured. Further all these parameters (rainfall, vibration, and moisture content) were used for monitoring and forecasting of slope failure, because of its utter importance in slope failure particularly in soil or unconsolidated debris. The water content variation for different rainfall intensity are measures with time as shown in the Figure 6. It is a known fact that most of the slope failures occur either during raining season or just after the rain. This is why we attempted to simulate and analyze the behavior of slope due to change in moisture content. Moisture content was analyzed as a critical parameter controlling the transient behavior of surface movements or slope failures.

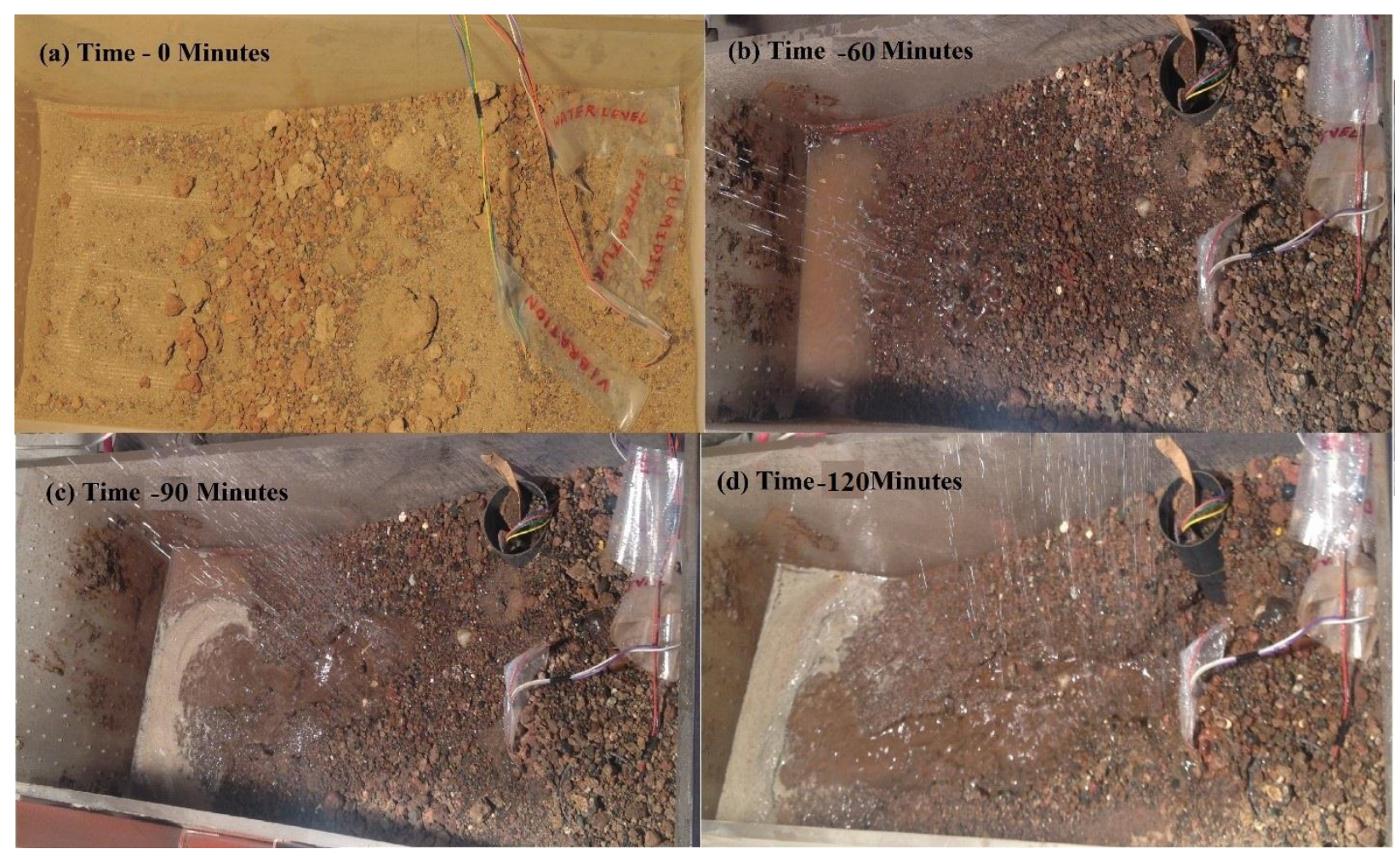

Figure 6. Analyzed slope through experiments performed with time (a). In dry condition, (b) when water poured with flow of $0.5 \mathrm{~mm} / \mathrm{min}$. (c) When water poured at $0.75 \mathrm{~mm} / \mathrm{min}$ (d) when water poured at $1 \mathrm{~mm} / \mathrm{min}$.

\subsection{Soil characterization}

The geotechnical parameters are experimented for the numerical simulation to check the stability of established slope. The unit weight of soil is characterized as per standard ASTM D7263. The angle of internal friction and the amount of cohesion are obtained as per standard ASTM D4767 and equal to $26.6^{\circ}$ and $1.15 \mathrm{KPa}$ for the dry condition of the soil, respectively. The unit weight, internal angle of friction and cohesion of representative soil samples are also experimented for different moisture content of soil to assess the numerical slope stability analysis, which is presented in the Table 2. 
Table 2. Soil parameter corresponding to water content.

\begin{tabular}{|c|c|c|c|c|}
\hline Moisture Content of Soil (\%) & $\begin{array}{l}\text { Unit } \\
\left(\mathbf{k N} / \mathbf{m}^{3}\right)\end{array}$ & Weight & Cohesion (KPa) & $\begin{array}{l}\text { Angle of Internal } \\
\text { Friction (Degree) }\end{array}$ \\
\hline $\operatorname{Dry}(0)$ & 18.0 & & 1.15 & 26.56 \\
\hline 10 & 19.8 & & 1.21 & 21.6 \\
\hline 15 & 21.5 & & 1.18 & 11.3 \\
\hline 20 & 23.4 & & 1.13 & 10.6 \\
\hline
\end{tabular}

\subsection{Numerical Simulation of Established Slope}

The similar slope of same dimension as in physical model are simulated with the help of LEM. The geotechnical parameters such as unit weight, cohesion, friction angle, tensile strength, Young's modulus and Poisson's ratio were considered for different moisture content as in Table 1. The computational domain of established slope geometry is shown in Figure 7.

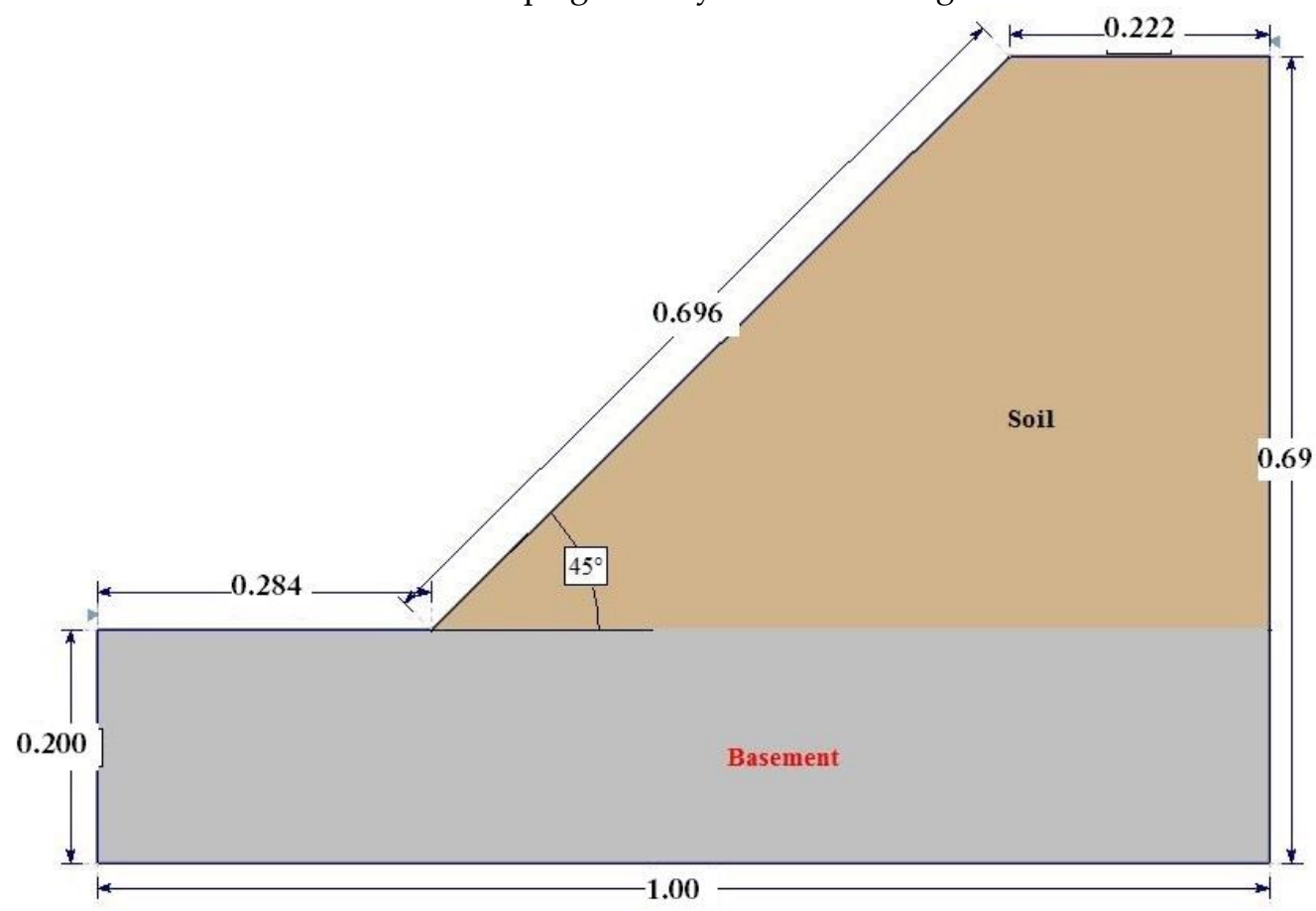

Figure 7. Geometry of the physical model.

The stability analysis using limit equilibrium method (LEM) was first accomplished with $0 \%$ moisture content, which gave a Factor of Safety (FoS) of 1.703 (Figure 8a). Further for precision, the slope was scrutinized at $10 \%, 15 \%$ \& $25 \%$ moisture content (Figure $8 b, 8 c \& 8 d$ ), that gave the FoS $1.493,1.070 \& 0.603$ respectively. The color variation of slip surface indicates the FoS along the slip surface. The results show that the critical slip surface occurred at the top of the slope. 

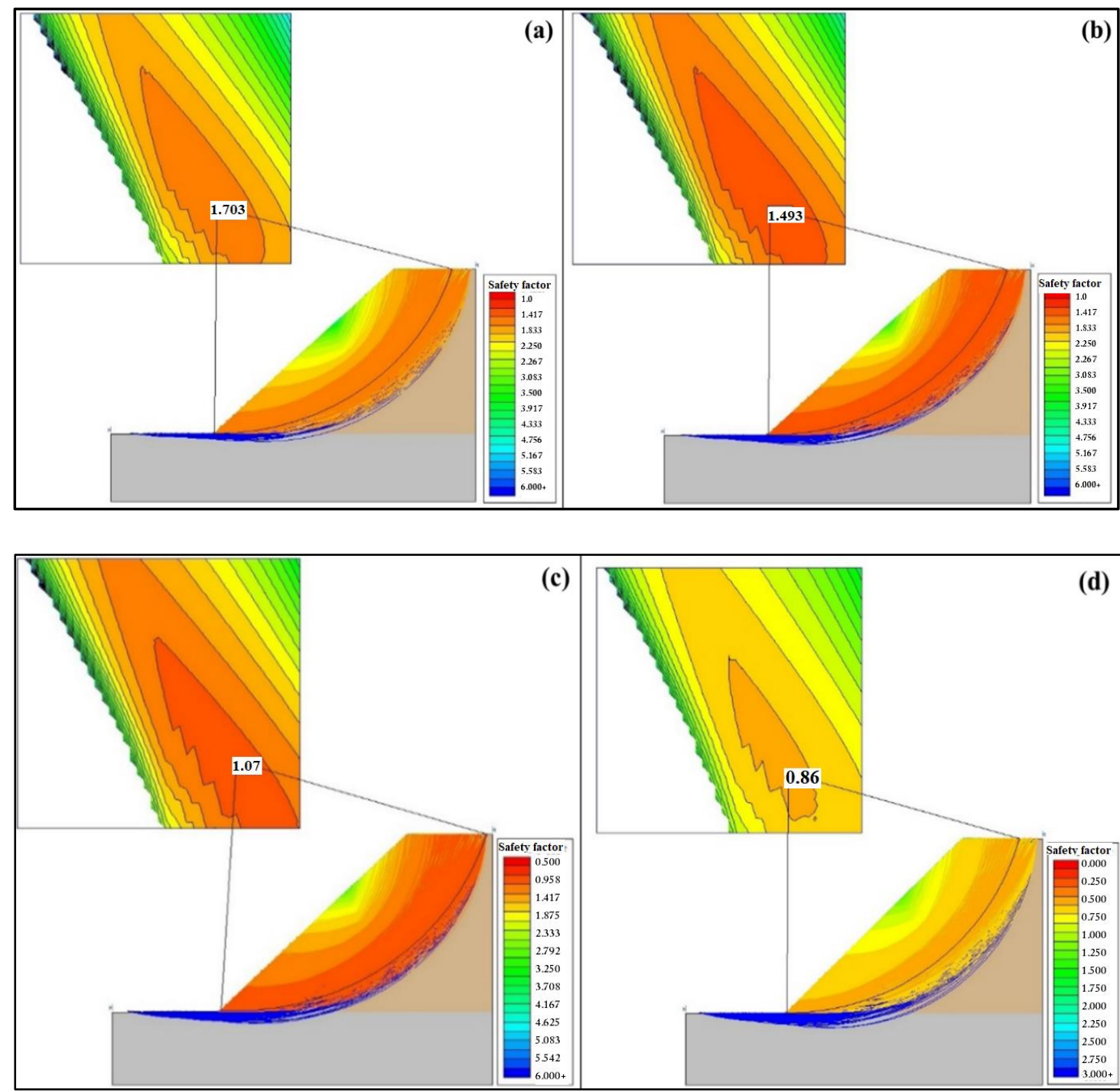

(c)

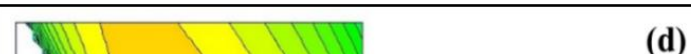

Figure 8. Analyzed slope by LEM, (a) FoS 1.70 at $0 \%$ moisture content (b) FoS 1.49 at $10 \%$ moisture content, (c) FoS 1.07 at 15\% moisture content and (d) FoS 0.60 at 20\% moisture content.

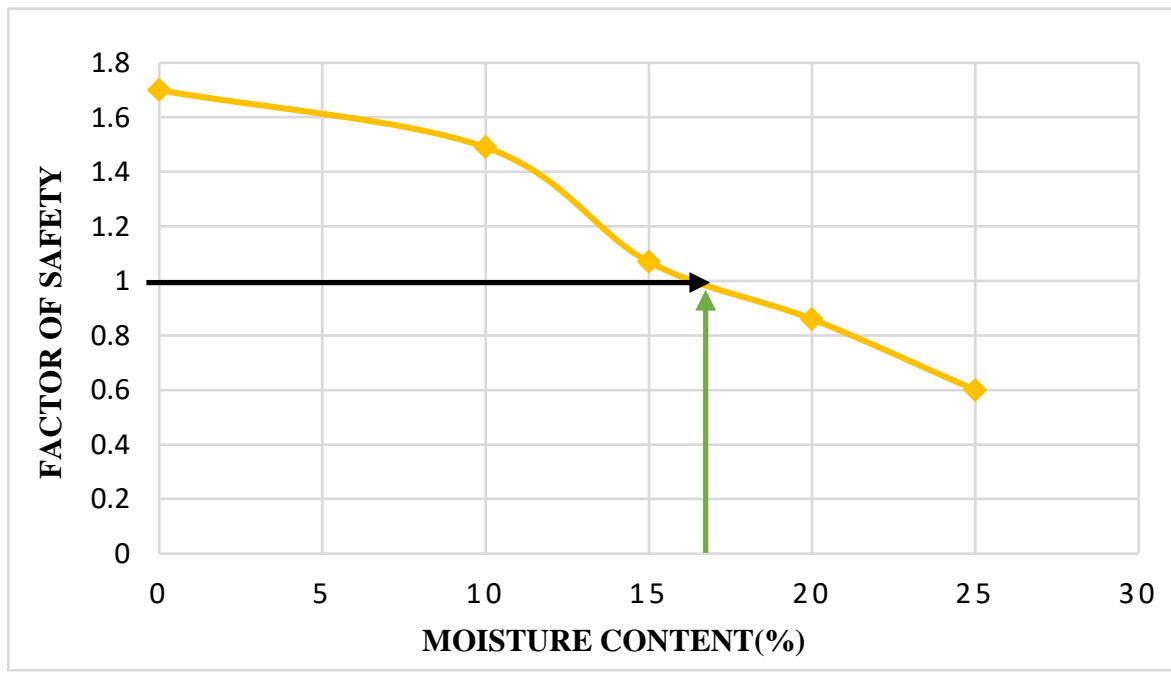

Figure 9. Variations in FoS of established slope. 


\subsection{Data used to assess applicability of rainfall thresholds}

Number of experiments were performed to assess the effectiveness of the present system and to observe the behavior of material failure at different water content. Rainfall data acquired from water level sensors has been taken into account to establish the threshold of rainfall and factor of safety. Four experiments are performed to analyze the slope stability at different water content.

Case. 1 Experiment performed in dry condition

Initially, experiment was performed at fully dry soil, i.e. $0 \%$ water content. The sensors were deployed to collect aforementioned parametric data continuously. This online data retrieval allowed monitoring the slope deformation continuously at a remote location. At dry condition, no change in any parameter can be perceived and data obtained from different sensors show constant value in the initial stage. At this stage no significant change in soil surface was observed.

The experiment started at time $t=0$ and at this time there is no change in the rainfall, moisture or acceleration till $t=30 \mathrm{~min}$ (Figure 9a). The soil sample was oven dried which made it free of any moisture. Figure 6a, clearly indicates that the water contain is zero value from the beginning till the end of 30 minutes. It reaffirms that a typical slope condition experiencing no rain and almost negligible initial moisture content caused very little or almost no perceivable displacement in slope. For this condition numerical simulation showed a FoS of 1.703, i.e. established slopes are in stable condition (Figure 8a)

Case. 2 Experiment performed water poured with flow of $(0.5 \mathrm{~mm} /$ minute $)$

While, in previous case, no deformation was seen because we have not given any input (no water poured), and the value obtained from the different sensors were also constant. Factor of safety was 1.703 for first case which shows slope is highly stable. In case 2, water is poured with a constant rate of $0.5 \mathrm{~mm} / \mathrm{min}$ from $\mathrm{t}=30 \mathrm{~min}$ to $\mathrm{t}=60 \mathrm{~min}$. which shows that the value of moisture content and acceleration are increasing gradually with water flow (Figure $6 \mathrm{~b}$ ). From $\mathrm{t}=30 \mathrm{~min}$. to $60 \mathrm{~min}$., the value of moisture content is increased from $0 \%$ to $10 \%$ and the value of acceleration is increased from $10 \mathrm{mg}$ from $12 \mathrm{mg}$, when value of rainfall is increased by $0 \mathrm{~mm}$ to $0.5 \mathrm{~mm} / \mathrm{min}$. Because of rain, fine particle started dislodging from the top of the slope. During this time span recorded factor of safety is 1.493 (Figure 8b). Which is lower in comparison with case 1 and also verify that the factor of safety is reduced with water.

Case. 3 Experiment performed water poured at $(0.75 \mathrm{~mm} / \mathrm{min})$

In case 2, when water was poured at rate of $0.5 \mathrm{~mm} / \mathrm{min}$, change is recorded in amplitude of moisture content, acceleration and factor safety is varying with amount of water content. While in case 3 , rate of water flow was increased by $0.5 \mathrm{~mm} / \mathrm{min}$ to $0.75 \mathrm{~mm} / \mathrm{mm}$ during time $\mathrm{t}=60 \mathrm{am}$ to $91 \mathrm{~min}$. Figure (6c). The value of moisture content increased by $10 \%$ to $15 \%$ and acceleration is increased by $12 \mathrm{mg}$ to $13.5 \mathrm{mg}$. This leads to movement of fine particle and small boulders started moving from upper and medium surface of the slope. Minor scouring can also be observed at the top face of the slope shown in Figure (6c). The simulated factor of safety for this case is 1.070, which clearly shows that slope is in critical condition (Figure 8c). 


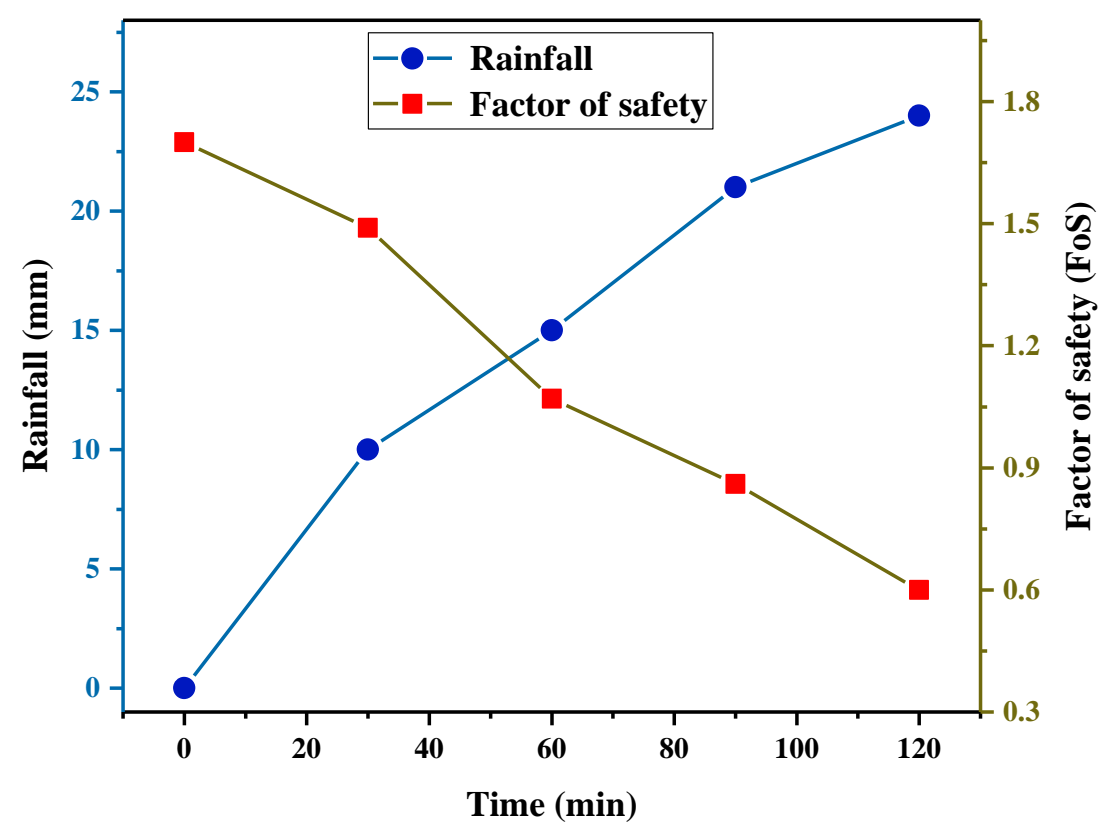

Figure 10. FoS and rainfall variations in study zone.

Case. 4 Experiment performed water poured at $(1 \mathrm{~mm} / \mathrm{min})$

In case 3, when water was poured at rate of $0.75 \mathrm{~mm} / \mathrm{min}$. The changes have been recorded in amplitude of moisture content, acceleration and factor safety which clearly demonstrate that factor of safety is decreasing with increase in water amplitude.

Whereas, in case 4 water flow rate has been kept at $1 \mathrm{~mm} / \mathrm{min}$ from $91 \mathrm{~min}$ to $120 \mathrm{~min}$. Figure. (6d). For this period of time moisture content increases from $15 \%$ to $20 \%$ and acceleration is also increased by $13.5 \mathrm{mg}$ to $14 \mathrm{mg}$ (Table 3). As a result of increase in moisture content and vibration associated with rainfall, major portion of slope material fails from upper and middle slope. Scouring can also be observed at face of top and middle slope due to high water flow (Figure 6d). This increase in pore water leads to loss of cohesive bonds in the soil mass. The soil mass mixes with water and flows downslope as a combined mass under the action of gravity. Factor of safety for this case is 0.63 which means slope is no more stable (Figure 8d).

Table 3. Factor of safety at different water content.

\begin{tabular}{llll}
\hline Rainfall (mm/min) & Moisture Content (\%) & Factor of safety & Acceleration $(\mathbf{m g})$ \\
\hline 0 & 0 & 1.703 & 10 \\
0.5 & 10 & 1.493 & 12 \\
0.75 & 15 & 1.070 & 13.5 \\
1 & 20 & 0.86 & 14 \\
\hline
\end{tabular}

\subsection{Threshold assessment for early landslide prediction}

In this section, a deterministic approach was used to decide the FoS thresholds for early warning of landslides disaster at laboratory scale experiment. While geo-mechanical properties taken into account during experiment to achieve the adequate response time essential to deal through natural disasters.

The simulation of rainfall pattern with FoS enable us to calculate total rainfall threshold for various soil slope as well as prototype development of small scale facility for rainfall threshold 
prediction for shallow landslides induced through heavy rainfall. Due to increase in rain intensity the pore water pressure increases in soil mass slope. The increase in pore water pressure reduce the normal stress along with the inclined plane. This reduces and further deteriorate the factor safety. The material failed along the weak plane. This result is further verified by movement recorded by sensor placed on the model.

As moisture content increases, factor of safety decreases due to increase in pore water pressure and loss of shear strength of the host material. Figure 9 demonstrate that the critical factor of safety was achieved at moisture content of $17 \%$, beyond which slope started failing at rapid rates. The total time taken by rainfall to drop factor of safety from 1.703 to 1.07 has been considered for the threshold establishment for landslides warning. Depends on the experiments conducted, number of threshold were tested which was between 1.203 and 1.07. The rainfall and FoS plot showed that with rise in rainfall content, the FoS is decreasing for the small scale physical model (Figure 10).

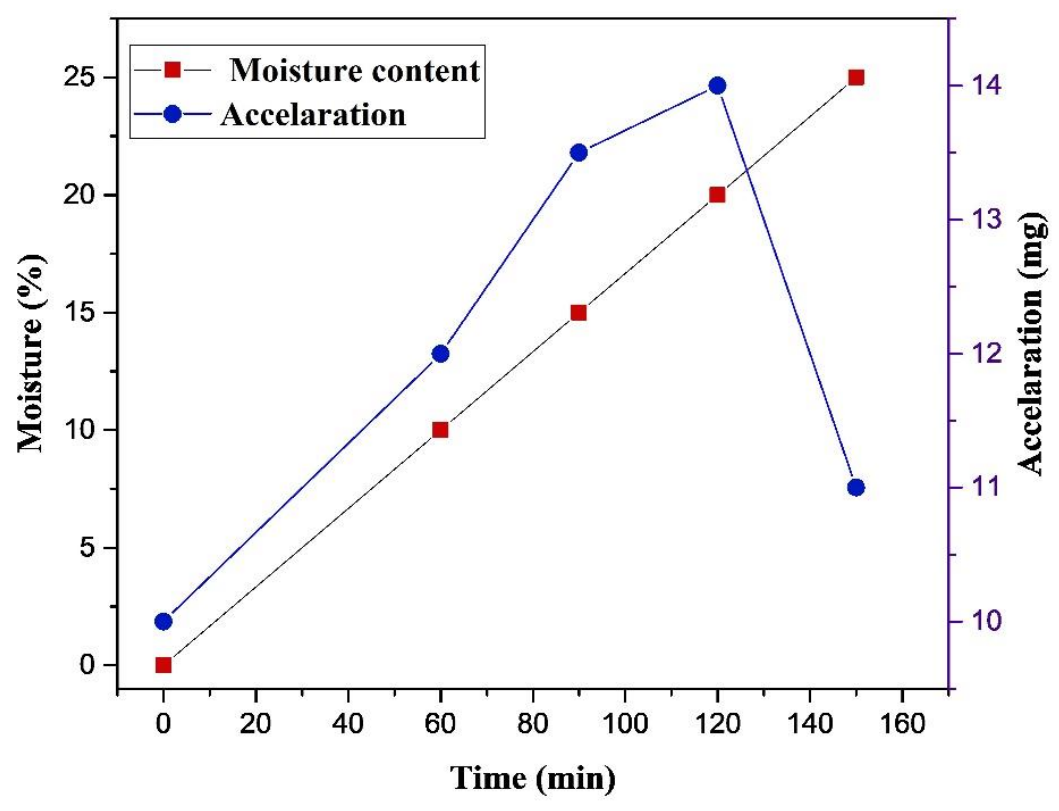

Figure 11. Acceleration (mg) and Moisture Content (\%) variations with time in Study Zone.

In the present study, total four no. of simple and effective parameters were used to calibrate the threshold, and a comparison has been made using data obtained from different set of experiments. Experimental data's shows that, with increasing rainfall content the moisture content is also increasing (Figure $9 \& 10$ ), and a threshold value of acceleration is achieved after a time span at particular moisture content (Figure 11).

\section{Conclusion}

Application of Wireless sensor network(WSN) for landslide monitoring and detection is one of the key research areas. This paper presents a small laboratory scale prototype deployment of WSN system for rainfall-based landslide monitoring and prediction. This technique uses sensor nodes integrated with different sensors, carries real time data rainfall data to the remote server. This research also described the real time slope rainfall threshold prediction and deformation characteristic of shallow landslides at laboratory scale deployment. Physical and numerical based experimental models have been simulated in different condition of slope to explain the deformation characteristic of shallow slope failure. Results found from various experiments demonstrate the surface movement and erosion of the slope mass. Results of numerical and physical model validate that the increasing rainfall, wind speed, particle size, slope angle and thickness of the particle size 
intensify the possibility of the initiation of slope failure. Slope angle is considered one of the crucial and critical parameter which control the slope stability.

Further, this laboratory level work would be scaled up to the prototype field deployment. The experiments would be performed to fix the effects of sensor node density and location of sensor node placement etc., for better prediction of rain fall induced landslides, which will help in the development and deployment of ultra-low cost wireless sensor network based early warning system for landslide prediction. To make a robust and reliable early warning system, deployment with increased spatial variability is in progress to detect slope movement for A full deployment with increased spatial variability, and the work in this regard is progressing to make a robust and reliable system to detect the movement in the slope in advanced for safety and stability.

Acknowledgments: The research work was carried out with the support from the Natural Resources and Data Management Systems Division (NRDMS), Department of Science and Technology, Ministry of Science and Technology, Government of India, New Delhi. The authors are grateful to NRDMS for the support and research grant (16DST004).

\section{References}

1. ASTM D4767-02 (2002) Standard test method for consolidated undrained triaxial compression test for cohesive soils. Annual book of ASTM standards. American Society for Testing and Materials, Philadelphia 2. ASTM D7263-09 (2009) Standard test methods for laboratory determination of density (unit weight) of soil specimen. Annual book of ASTM standards. American Society for Testing and Materials, Philadelphia 3. Bishop, A.W., "The use of the slip circle in the stability analysis of earth slopes." Geotechnique, 5 (1), 7 $-17,1955$.

4. C. Lepore, E. Arnone, L.V. Noto, G. Sivandran, R.L. Bras, Physically based modeling of rainfalltriggered landslides: a case study in the Luquillo forest, Puerto Rico. Hydrology and Earth System Sciences, 17(9) (2013) 3371-3387.

5. D. Collins, Brian \& Znidarcic, Dobroslav. (2004). Stability Analyses of Rainfall Induced Landslides. Journal of Geotechnical and Geoenvironmental Engineering - J GEOTECH GEOENVIRON ENG. 130. 10.1061/(ASCE)1090-0241(2004)130:4(362).

6. Rahardjo, H \& W. Li, X \& Toll, David \& Leong, E. (2001). The effect of antecedent rainfall on slope stability. Geotechnical and Geological Engineering. 19. 371-399. 10.1023/A:1013129725263.

7. H.R. Bogena, J.A. Huisman, C. Oberdörster, H. Vereecken, Evaluation of a low-cost soil water content sensor for wireless network applications, Journal of Hydrology, 344(1) (2007) 32-42.

8. Huisman, J.A., S.S. Hubbard, J.D. Redman, and P.A. Annan. 2003. Measuring soil water content with ground penetrating radar: A review. Vadose Zone J. 2:476-491.doi:10.2136/vzj2003.4760

9. J. Corominas, C. Van Westen, P. Frattini, L. Cascini, J.P. Malet, S. Fotopoulou, F. Catani, M. Van Den Eeckhaut, O. Mavrouli, F. Agliardi, K. Pitilakis, Recommendations for the quantitative analysis of landslide risk. Bulletin of engineering geology and the environment, 73(2) (2014), 209-263.

10. L. K. Sharma, Ravi Kumar Umrao, Rajesh Singh, M. Ahmad, T. N. Singh, Stability Investigation of Hill Cut Soil Slopes along National Highway 222 at Malshej Ghat, Maharashtra, Journal Geological Society of India 89(2) (2017) 165-174

11. L. Kuriakose, Sekhar \& Sankar, G \& Muraleedharan, C. (2008). History of landslide susceptibility and a chorology of landslide-prone areas in the Western Ghats of Kerala, India. Environmental Geology. 57. 1553-1568. 10.1007/s00254-008-1431-9.

12. L. Montrasio, L. Schiliro, A. Terrone, Physical and numerical modelling of shallow landslides, Landslides, 13(5) (2015) 873-883.

13. Lee, Jin-Shyan, and Yuan-Ming Wang. "Experimental evaluation of ZigBee-based wireless networks in indoor environments." Journal of Engineering 2013 (2013).

14. P. Bláha, K. Müller, Geophysical monitoring as an information source of rock massif behaviour. Acta Montanistica Slovaca, 13(2) (2008) 223-228.

15. P. Zhou and L. Yao, Landslide hazard early warning system in China and future trends. In IAEG, (2006) $1-5$.

16. R. Trivedi, V. Vishal, S. P. Pradhan, T. N. Singh, J. C. Jhanwar, Slope stability analysis in limestone mines, International Journal of Earth Sciences and Engineering, 5 (4) (2012) 759-766. 
17. R.L. Baum, D.L. Galloway, E.L. Harp, Landslide and land subsidence hazards to pipelines (No. 20081164). Geological Survey (US) (2008).

18. S. P. Pradhan, V. Vishal, T. N. Singh, V. K. Singh, Optimisation of dump slope geometry vis-à-vis flyash utilisation using numerical simulation, American Journal of Mining and Metallurgy, 2 (1) (2014) 1-7.

19. S. S. Gupte, R. Singh, V. Vishal, T. N. Singh, Detail investigation of stability of in-pit dump slope and its capacity optimization, International Journal of Earth Sciences and Engineering, 6 (2) (2013) 146 - 159.

20. S.K. Shukla, S.K. Chaulya, R. Mandal, B. Kumar, P. Ranjan, P.K. Mishra, G.M. Prasad, S. Dutta, V. Priya, S. Rath, K. Buragohain, Real-Time Monitoring System for Landslide Prediction Using Wireless Sensor Networks, International Journal of Modern Communication Technologies and Research, 14(12) (2014) 1419.

21. T.N. Singh, R. Singh, B. Singh, L.K. Sharma, R. Singh, M.K. Ansari, Investigations and stability analyses of Malin village landslide of Pune district, Maharashtra, India. Natural Hazards 81 (2016), 2019-2030

22. V. Ramesh, Design, development, and deployment of a wireless sensor network for detection of landslides Ad Hoc Networks 13 (2014) 2-18.

23. V. Vishal, T. Siddique, R. Purohit, M. K. Phophliya, S. P. Pradhan, Hazard assessment in rockfall prone Himalayan slopes along National Highway - 58, India: Rating and Simulation, Natural Hazards, 85 (2017) 487-503.

24. V. Vishal, S. P. Pradhan, T. N. Singh, an investigation on stability of mine slopes using two dimensional numerical modeling, Journal of Rock Mechanics \& Tunnelling Technology, 21 (1) (2015) 49-56.

25. V.J. Hodge, S. O'Keefe, M. Weeks, A. Moulds, Wireless sensor networks for condition monitoring in the railway industry: A survey. IEEE Transactions on Intelligent Transportation Systems, 16(3) (2015) 10881106.

26. Y. Gui, Z.G. Tao, C.J. Wang, X. Xie, Study on remote monitoring system for landslide hazard based on wireless sensor network and its application. Journal of Coal Science and Engineering (China), 17(4) (2011), 464-468. 